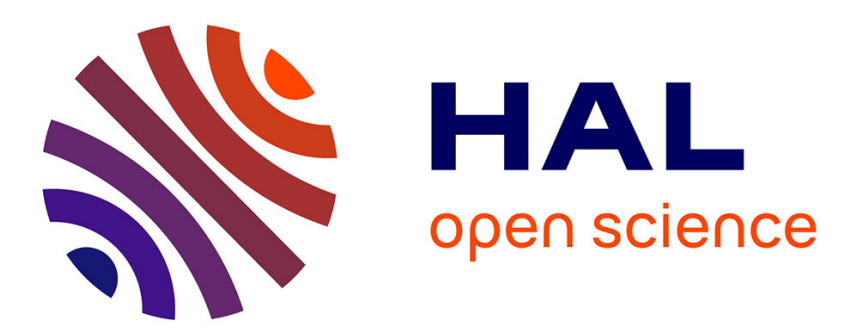

\title{
Réflexions politiques et éthiques contre la possibilité d'une autonomisation radicale des communs
}

Pierre Crétois, Caroline Guibet Lafaye

\section{To cite this version:}

Pierre Crétois, Caroline Guibet Lafaye. Réflexions politiques et éthiques contre la possibilité d'une autonomisation radicale des communs. Éthique publique, 2016, 17 (2). halshs-01311029

\section{HAL Id: halshs-01311029 \\ https://shs.hal.science/halshs-01311029}

Submitted on 25 May 2017

HAL is a multi-disciplinary open access archive for the deposit and dissemination of scientific research documents, whether they are published or not. The documents may come from teaching and research institutions in France or abroad, or from public or private research centers.
L'archive ouverte pluridisciplinaire HAL, est destinée au dépôt et à la diffusion de documents scientifiques de niveau recherche, publiés ou non, émanant des établissements d'enseignement et de recherche français ou étrangers, des laboratoires publics ou privés. 
Réflexions politiques et éthiques contre la possibilité d'une autonomisation radicale des communs

Pierre Crétois, docteur en philosophie, mène des recherches sur l'histoire du droit de propriété et les transformations actuelles des régimes d'appropriation dans le cadre du Sophiapol (EA 3932).

Caroline Guibet Lafaye est directrice de recherche au CNRS (Centre Maurice Halbwachs - EHESS - ENS). Agrégée et docteur en philosophie de l'Université de Paris-I Panthéon-Sorbonne, elle consacre ses travaux à l'analyse de l'appréciation des inégalités et de leur légitimation, en contexte redistributif. Elle s'appuie sur un corpus normatif philosophique et économique ainsi que sur ses investigations en sociologie pour cerner les conditions d'acceptabilité de mesures redistributives et de principes d'allocation des biens. Caroline Guibet Lafaye est l'auteur de plusieurs articles et ouvrages dans ces domaines, dont deux livres parus en 2006 aux Presses universitaires de Laval : La justice comme composante de la vie bonne et Justice sociale et éthique individuelle, et un en 2012, Le juste et l'inacceptable. Les sentiments d'injustice contemporains et leurs raisons (PUPS). Elle a également fait paraître en 2009 Penser le bonheur aujourd'hui, aux Presses universitaires de Louvain.

Résumé : La référence aux communs constitue aujourd'hui une étiquette désignant une alternative à la société de marché. Le problème que nous aborderons dans cet article porte précisément sur le statut des boucles de contre-réaction qu'une société s'impose à elle-même et par lesquelles les membres des groupes partiels trouvent, dans une instance extérieure, un recours juridique. Nous essaierons de montrer que l'approche par les communs ne permet pas de se dispenser d'un tiers envisagé comme recours arbitral n'appartenant pas aux communautés.

Mots clefs : communs; philosophie; régulation; domination; liberté individuelle

\begin{abstract}
Nowadays, the reference to common is a label designating an alternative solution to the market society. The problem that we deal with in this article is about the status of feedback loops that human societies requires against its functioning biases and through which members of partial groups of this society find an external legal recourse. We try to show that the common approach isn't self-sufficient and suppose the intervention of an external institution which should be enabled to arbitrate conflicts between communities and their members.
\end{abstract}

Key words: commons; philosophy; regulation; domination; individual freedom

La référence aux communs ${ }^{1}$ constitue aujourd'hui soit une étiquette désignant une alternative à la société de marché (pour ceux qui défendent la conception la plus

\footnotetext{
${ }^{1}$ Qu'il nous soit permis de remercier Isabelle Aubert, Sébastien Broca et Stéphane Haber pour leur relecture et leurs suggestions.
} 
compréhensive des communs ${ }^{2}$ ), soit seulement un régime spécifique de gestion des biens qui se déploie en parallèle de l'appropriation privative et ne la remet pas en cause ${ }^{3}$. La transition vers une société marquée par des formes communautaires d'appropriation plutôt que par la propriété privée est appelée par des motifs anthropologiques (contestation de l'individualisme intéressé, revendication d'une vie humaine fondée sur le partage et l'appartenance), par des motifs économiques (contestation des rapports de production, fondés sur l'appropriation privative et la marchandisation des ressources) et, enfin, par des motifs politiques (promotion de la démocratie et de l'autogouvernement à tous les niveaux et dans tous les domaines, y compris productifs). Cette proposition inclut positivement un programme de réforme des régimes d'appropriation pour les rendre plus inclusifs et participatifs, la promotion des solidarités sociales et des prises de décision démocratique entre toutes les parties prenantes.

Un commun désigne tout objet investi par l'activité productive et/ou décisionnelle d'un groupe de personnes. Ceux des partisans des biens communs qui s'affranchissent de la typologie de Samuelson admettent que la définition d'un commun ne provient pas de ses caractéristiques objectives, mais qu'il s'agit d'un arrangement social spécifique qui résulte principalement de la subordination d'une ressource à un groupe d'individus, qui la gouverne ensemble. Ces ressources sont multiples et peuvent aller des connaissances aux ressources environnementales, en passant par des savoirs industriels et des moyens de production.

L'hypothèse du primat communautaire pour définir les communs tient, à notre sens, à l'usage un peu naïf de la notion de communauté par certains de ses théoriciens (non pas tous) et à la minimisation des dissonances (parfois des conflits) internes inhérents à leur gestion ${ }^{4}$. La primauté accordée à la communauté dans la définition des communs pose pourtant la question de son emprise sur ses membres. Ainsi, notre propos sera de critiquer l'optimisme peut-être naïf - de certains défenseurs des communs (invoquant parfois les communs médiévaux comme modèle alors qu'ils furent loin d'être idéaux pour ce qui concerne la protection des personnes) et les questions qu'il conduit à occulter. Pour cela, il ne faut pas sous-estimer la difficulté que représente la définition des processus de décision collectifs au sein des communautés productives ni négliger les risques de violences portées aux individus.

Ainsi, Jean-Marie Harribey soulignait dans le numéro de l'hiver 2015 de la revue Les possibles:

Cependant, si le commun n'est pas donné dans l'être du social ni même inscrit en lui à titre de "tendance" qu'il suffirait de stimuler, c'est parce qu'il est d'abord et avant tout une question de droit, donc de détermination de ce qui doit être, il faut donc se demander qui peut et qui doit dire le droit. Qui, sinon une entité au-dessus de la petite communauté gérant un commun ? Qui fera respecter ce droit dans les sociétés modernes? Les petites communautés auraient-elles leurs propres tribunaux ? Est-il sûr que seules les pratiques sociales indépendantes de l'État sont susceptibles de "créer du droit"? (Harribey, 2015)

Même si cette conception un peu radicale d'une croyance dans l'auto-organisation partagée par la plupart des théoriciens des communs est rarement défendue à l'état pur, elle apparait, la plupart du temps, comme un présupposé prégnant, sous-jacent à la promotion des formes de gestion en commun des ressources. Ainsi récemment des mouvements pour demander la reconnaissance et l'encadrement juridique des communs, notamment numériques, par l'État ont vu le jour (Couture, 2013). En outre, certains théoriciens comme Elinor Ostrom donnent

\footnotetext{
${ }^{2}$ Voir notamment l'approche de Pierre Dardot et Christian Laval qui voient dans les communs une méthodologie politique générale (Dardot et Laval, 2014).

${ }^{3}$ Les économistes préfèrent cette approche, c'est notamment le cas d'Elinor Ostrom pour qui les biens communs sont une catégorie de bien spécifique distincte des biens privés, de club et publics, conformément à la classification de Samuelson.

${ }_{4}$ «L'absence, ou au minimum la sous-estimation du rôle des logiques de pouvoir, est une des faiblesses du type d'analyse proposée par Elinor Ostrom, alors même que son approche est de nature à permettre précisément une certaine manière d'explorer cette question » (Wenstein, $2015: 83$ ).
} 
un rôle à l'État comme tiers régulateur (Ostrom, 1990 ; Holland, 2010) ${ }^{5}$. Néanmoins le rôle de l'État n'est pas suffisamment repensé dans le cadre des communs, admettant des formes renforcées d'auto-organisation décentralisée et redéfinissant, dans ce cadre, les conditions de la protection de la liberté des personnes.

En effet, si la capacité décisionnaire de la communauté est accrue, cela lui donne plus de pouvoir. Elle peut alors plus facilement interférer arbitrairement sur les choix libres des individus, même si elle ne le fait pas toujours. Si c'est le cas, comment garantir la «nondomination », c'est-à-dire un statut qui confère aux individus une immunité, y compris contre les exigences arbitraires du groupe auquel ils appartiennent ${ }^{6}$. Or, la fonction du tiers arbitre impartial et raisonnable n'est pas toujours suffisamment pensée dans l'approche des communs qui reste, parfois, prise dans l'image idéalisée de petites communautés locales autonomes, présentées comme autogouvernées et productrices de décisions incontestables. Tel est le point faible ou le biais un peu naïf de certains théoriciens des communs. Cela nous conduira à revenir, dans un premier temps, sur deux hypothèses importantes dans le débat actuel, puis sur la fonction d'une tierce partie pour arbitrer les conflits entre la communauté et ses membres ${ }^{7}$.

\section{Deux approches pour défendre le primat de la communauté en tant que définition d'un commun}

Deux manières d'aborder la question du commun prévalent, qu'elles reposent sur l'étude des régimes d'appropriation collectifs (Ostrom, 1990) ou sur celle des praxis collectives à l'origine des modes de gouvernance commune sans appropriation (Dardot et Laval, 2014). La version ostromienne des communs s'appuie sur l'idée anti-hardinienne (Hardin, 1968) que des modes d'appropriation collectifs n'impliquent pas nécessairement de conséquences économiques sous-optimales. La version de Dardot et Laval fait fond sur l'idée, antiutilitariste, qu'une société ne saurait se réduire au résultat d'un calcul d'intérêt. Nous allons montrer que ces deux approches, parce qu'elles sous-estiment les rapports de force et les conflits possibles, au sein des communautés, défendent une vision idyllique de la communauté souvent passéiste.

\section{L'approche ostromienne : des régimes d'appropriation alternatifs}

L'un des présupposés de l'économie classique est que les biens privés représentent, dans la plupart des cas, la forme optimale de l'attribution des ressources. Les biens communs, rivaux mais non exclusifs selon la classification de Samuelson (1954), seraient destinés à n'être pas entretenus à moins d'être assujettis à des formes d'appropriation privées ou publiques, responsables de leur gestion. C'est à Garrett Hardin que nous en devons la démonstration. L'argument de Hardin (1968) est simple. Il tient au fait que dans le cas d'un pâturage laissé en commun entre plusieurs bergers, chacun, en ajoutant une bête, gagne un point d'utilité, alors que la perte étant partagée entre les bergers, il ne perd qu'une portion

\footnotetext{
5 Les deux dernières règles d'organisation des communs portent sur leur rapport à l'État: l'État doit reconnaître l'organisation en place ; l'ensemble du système est organisé à plusieurs niveaux.

${ }^{6}$ La notion de non-domination a été élaborée par Philip Pettit pour désigner le fait de bénéficier d'un statut légalement garanti qui prémunit durablement contre toute interférence arbitraire d'un tiers : «l'absence de domination signifie que l'on jouit d'une absence d'interférences imputables à des pouvoirs arbitraires, non pas seulement dans le monde réel, mais dans l'ensemble des mondes possibles [...]. » (Pettit, $2004: 44-45)$

${ }^{7}$ Notre proposition est, en un sens, proche de celle de Dagan et Heller (2001) dans leur article The liberal commons, bien que leur position consiste davantage à montrer la compatibilité entre le libéralisme et les formes communautaires d'autoorganisation. Nous cherchons davantage à insister sur un impensé propre à une approche trop enthousiaste et radicale du recours à l'auto-organisation. Notre propos vise à souligner le fait que, sauf à abandonner certains acquis essentiels de la modernité politique, cette dernière ne saurait se passer du recours à un tiers arbitre.
} 
d'un point d'utilité. Pour lui, le calcul est rapidement fait : le berger a intérêt à accroître son troupeau autant qu'il le peut puisque le coût de chaque bête se trouve dilué. Néanmoins, si l'on suit ce raisonnement, la destruction du champ est inéluctable. Indépendamment de l'anthropologie simpliste qui sous-tend l'approche d'Hardin, celle-ci ouvre, en l'excluant par principe, un nouveau champ d'études : celui des régimes d'appropriation et des modes de gouvernance des biens communs non assujettis au régime de la propriété privée. Il est impossible, dans le cadre de la présente analyse, de rentrer dans le détail de la proposition maintenant bien connue d'Elinor Ostrom. Nous nous contenterons donc de renvoyer aux travaux de Benjamin Coriat (2015a et 2015b) et Fabienne Orsi (2015) qui en constituent une exégèse précise et abondante.

La principale thèse d'Elinor Ostrom consiste à montrer qu'il est possible de posséder une ressource en commun, notamment ce qu'elle appelle les Pools communs de ressource (CPR), d'une manière qui ne conduise pas à sa perte. Elinor Ostrom et Edela Schlager (1992) proposent, pour ce faire, de distribuer des droits d'utilisation (droit d'accès, droit de prélèvement) et des droits d'administration (droit de gestion, droit d'exclusion, droit de vendre).

Les deux points principaux qui permettent à Elinor Ostrom de contester Hardin tiennent, en premier lieu, au fait de penser le bien commun à travers des droits distribués évitant de faire équivaloir bien commun et bien laissé libre puisque, ainsi pensé, le bien commun est inscrit dans une forme d'appropriation réglée. Le régime d'appropriation distributif permet alors une gestion alternative au régime d'appropriation centralisé, typique du droit de propriété. Ce régime d'appropriation alternatif permettrait de régler les rapports sociaux quant aux ressources, c'est-à-dire d'éviter de les laisser à l'arbitraire des décisions et des calculs d'intérêt individuels dont résulterait la perte, selon l'hypothèse d'Hardin.

Le second point réside dans l'existence, en plus des droits opérationnels ou des droits d'utilisation, d'un droit de gestion ou de gouvernance de la ressource qui empêche qu'elle soit laissée aux seules décisions isolées des individus. Ce droit constitue d'ailleurs un élément essentiel de la définition du commun. Par ce moyen, il devient possible de coordonner les intérêts des agents de façon immanente, c'est-à-dire à partir d'une décision délibérée des agents eux-mêmes. Aussi dans ce cadre, pour qu'il y ait un commun, il faut à la fois une ressource commune, un système de droits distribués et un principe de gouvernance commune.

Elinor Ostrom s'appuie sur l'étude de communautés traditionnelles. Cette référence est également partagée par Benjamin Coriat (2014 et 2015) qui évoque les communs d'Ancien Régime. Dans ces communautés, les droits sont en effet distribués et coexistent parfois avec des modes de gouvernance communs. Cependant, le problème tient alors au rapport des individus à la communauté à laquelle ils appartiennent. Ces risques sont liés à l'extension du pouvoir de gouvernance, en tant qu'il peut limiter ou restreindre les droits des individus. Cette marge opérationnelle des décisions collectives les rend susceptibles d'être source de domination ou bien, au contraire, d'en préserver les individus. Il s'agirait, selon nous, de compléter ou de corriger l'interprétation courante de l'approche ostromienne, en la matière, par la limitation a priori de l'extension du pouvoir décisionnel de la communauté, au nom des droits individuels, et donc par l'introduction d'un principe de limitation transcendant la petite communauté de producteurs et s'imposant à elle de l'extérieur. Une telle hypothèse impliquerait l'introduction d'un ensemble minimal de normes non immanentes. Dans les communautés traditionnelles, en effet, le poids du collectif peut parfois revêtir des formes oppressives pour l'individu. Dans certaines communautés villageoises médiévales, comme en Bigorre, le nouvel arrivant n'acquérait le droit de voisinage qu'après avoir fait ses preuves durant un an sous peine d'exclusion du village (Follain, 2008). On peut citer aussi le droit que les communautés villageoises s'attribuaient sur les lopins exploités comme le droit de première herbe, le droit de parcours ou le droit de vaine pâture (Crétois, 2015a). Si l'on ajoute 
à cela la possibilité qu'existent des asymétries dans les délibérations (si celles-ci ne sont soumises à aucune métarègle) et que l'égalité des agents n'existe pas (du fait que certains peuvent être dotés de droits sur les ressources dont les autres sont exclus, mais aussi de dons et de capacités asymétriques), l'approche par les droits distribués est loin de préserver l'égalité des individus ainsi que des formes de démocratie, compatibles avec la protection des individus contre la domination.

La plupart des auteurs abordant les communs sous cet angle font preuve d'un - trop grand optimisme à l'égard du pouvoir auto-organisationnel des communautés. Si cette vision qui fait de l'approche par les communs une troisième voie entre l'État et le marché n'est pas le fait d'Elinor Ostrom, qui défendait une vision plus complexe et modulaire, intégrant des formes de régulation étatiques (Chanteau et Labrousse, 2013), elle est néanmoins répandue parmi ceux qui s'en réclament. Or, une telle confiance occulte des difficultés évidentes.

\section{L'approche de Dardot et Laval : un primat de la praxis collective dans la définition des communs}

L'approche concurrente de celle précédemment exposée tend à affirmer qu'un commun n'est pas une chose, une substance, qu'il n'est pas plus le résultat des interactions des individus. Le commun constitue plutôt le résultat d'une praxis collective. Cette approche fait signe vers des formes de démocratie radicale, au sein desquelles la distribution des droits aux individus est secondaire et ne saurait être opposée au droit de la communauté. Pour Dardot et Laval (2014), la question n'est nullement celle des modes d'appropriation alternatifs, mais plutôt celle de la subordination de l'usage des biens aux finalités définies par la communauté. Pour cette raison, ces auteurs évoquent l' « institution de l'inappropriable ». Celle-ci se définit à travers de simples droits d'usages, distribués en fonction des conditions résultant de la praxis collective. Le collectif auto-organisé n'est lui-même pas tant propriétaire que régulateur de l'usage des ressources par ses membres.

Une autre interprétation du commun très proche de celle-ci s'appuie sur une attitude théorique que l'on pourrait qualifier d'anti-utilitariste. Elle est également mobilisée par François Flahault (2011) ou d'une manière moins explicite par Alain Caillé (2011) ou JeanClaude Michéa (2007) ${ }^{8}$. Dans cette perspective, on ne saurait considérer que les individus se font seuls. La société ne saurait être non plus le résultat d'un calcul d'intérêts des individus puisque ce qui fait fondamentalement leur être-en-commun est antérieur à tout calcul et ne saurait donc s'y réduire. Cette interprétation est nourrie par la récusation de l'anthropologie de l'économie classique qui accepte comme hypothèse fondamentale l'existence d'un individu atomique porteur d'un intérêt propre. C'est ce que Marx a caractérisé comme une robinsonnade (Chappé et Crétois, 2014). Une perspective alternative consiste au contraire à défendre que ce que chacun est et ce que chacun a dépend d'une appartenance à une communauté humaine, de telle sorte que notre singularité n'est aucunement indépendante de cette appartenance.

Dans ce cadre, la proposition institutionnelle consiste à refuser le principe même de l'appropriation pour promouvoir l' « institution de l'inappropriable » (Guibet Lafaye, 2016) et la subordination des ressources aux finalités définies en commun :

\footnotetext{
${ }^{8}$ C'est, selon Michéa, contre l'être-en-commun fondamental que l'individualisme libéral s'est constitué : «le souci initial des libéraux n'était-il pas avant tout, de bannir de la vie politique les seules références à une conception commune de la morale et de la vie bonne? Une telle position n'exigeait par elle-même que la simple privatisation des valeurs morales, religieuses ou philosophiques, et nullement leur abolition. » (Michéa, 2007 : 129). Sa référence est la théorie du don de Mauss, qui « implique la primauté du cycle ou de la relation sur les individus eux-mêmes [...] obligeant ainsi à inscrire au cœur du sujet humain lui-même cette dimension d'endettement symbolique qui constitue l'un des fondements essentiels de son incomplétude constituante » (Michéa, 2007 : 133-134).
} 
Il s'agit de problématiser l'approche en termes de propriété commune pour lui substituer la perspective de l'institution d'un droit d'usage du commun : il n'y a pas de droit du commun parce que la communauté comme personne morale est le sujet titulaire de la propriété, mais par l'institution d'un droit d'usage collectif et de la destination commune de cet usage collectif. Par exemple, si l'on veut rendre une place publique "commune", en empêchant qu'elle puisse être cédée par l'État à des entrepreneurs privés, il faut garantir à chaque habitant de la ville un droit d'usage de cette place pour autant que cet usage ait lui-même pour finalité la possibilité de tout un chacun de l'utiliser. À l'inverse, la propriété commune en tant que propriété de la communauté peut très bien être exclusive en laissant à l'extérieur ceux qui ne lui appartiennent pas, de sorte que sa finalité peut n'être pas commune. C'est la raison pour laquelle Dardot et Laval identifient la finalité du commun à "l'inappropriable". (Sauvêtre, 2015)

Une telle approche semble être compatible avec le respect des personnes, puisque « commun » peut être pris en un sens distributif comme c'est le cas dans l'exemple de la place publique dont jouit chacun des usagers sans que personne n'ait le droit de l'en exclure. Cependant, (a) qui décide du caractère distributif ou collectif du sujet bénéficiaire sinon la communauté elle-même ? Celle-ci se trouve investie d'une capacité à déterminer les règles de gouvernance des ressources, y compris de les subordonner à des finalités contraires à l'accomplissement des individus, au nom de leur intégration dans la communauté. De même, (b) qui décide de l'omnipotence de la communauté délibérante et de la réduction de toute considération juridique à des considérations politiques et à des procédures de démocratie radicale, jamais explicitement fixées, qui assignent toujours l'individu à sa destination au sein d'une communauté ?

Dardot et Laval (2013) ou Michéa (2007) prennent pour modèles les mouvements ouvriers du XIX ${ }^{\mathrm{e}}$ siècle et les approches spontanéistes. Or, il est évident que le monde ouvrier du XIX ${ }^{\mathrm{e}}$ siècle ou les formes d'auto-organisation où la collectivité délibérante distribue les tâches ne doit pas gommer la présence de conflits parfois violents en son sein, où le rapport de force est, in fine, le principe de la décision, ou bien la dissolution de l'individu dans le collectif auquel l'individu appartient. Ces aspects sont incompatibles avec certaines des attentes contemporaines les plus essentielles, en termes de préservation de la liberté individuelle. La question porte alors aussi bien sur les conditions de respect du pluralisme et de l'égalité délibérative des agents que sur l'existence d'un recours extérieur pour l'individu contre la communauté, c'est-à-dire une clause de limitation du pouvoir de la communauté susceptible d'arbitrer les différents éventuels entre la communauté et l'un de ses membres. Nous retrouvons ici le même type de limites que celles rencontrées dans l'approche ostromienne.

Dans les deux approches évoquées, les problèmes sont identiques. La référence quasi idyllique aux communautés traditionnelles revêt une certaine pertinence, dans la mesure où le monde économique contemporain s'est accompagné de la dissolution des solidarités sociales traditionnelles. Néanmoins, il convient de ne pas négliger le type d'avantages que l'individualisme et le pluralisme de formes politiques contemporaines procurent (Picavet, 2011). Comment donc parvenir à réintroduire des formes d'être-en-commun qui n'obèrent pas les acquis positifs du pluralisme et de l'individualisme? Telles qu'elles sont aujourd'hui formulées, les théories des communs présentent trois difficultés : (a) la première concerne les conditions susceptibles de tempérer la pression toujours possible de la communauté sur ses membres; (b) la deuxième porte sur les conditions permettant de garantir le caractère démocratique du résultat de la délibération et son respect de l'exigence d'émancipation humaine, caractéristique de la modernité ; (c) la délimitation de la communauté porteuse de droits (notamment en tant qu'elle dispose d'un pouvoir potentiellement arbitraire d'inclure ou d'exclure). La préservation de ces conditions suppose l'existence et l'intervention d'un tiers 
extérieur susceptible de servir de médiateur entre l'individu et la communauté à laquelle il appartient et capable de promouvoir de nouveaux droits sociaux garantissant, à tous les individus, l'accès le plus large aux conditions d'un «projet rationnel de vie » (Rawls, 1971). C'est peut-être ainsi qu'il serait possible de définir l'État partenaire dont parle Michel Bauwens (2015) ou la fonction de l'État, évoquée par Ostrom dans les huit règles qu'elle propose (Ostrom, 1990).

\section{Le recours externe à l'État comme contrôle extérieur à l'auto- organisation des communs}

Nous nous proposons, dans cette partie, d'établir les linéaments d'une réflexion sur le rôle d'un État qui aille au-delà des règles 7 et 8 proposées par Ostrom (1990). Cette dernière propose, en effet, que l'État reconnaisse juridiquement le droit à l'auto-organisation (c'est dans ce sens que vont, par exemple, certains débats et certaines avancées actuelles autour des logiciels libres et des communautés organisées autour d'eux [Couture, 2010]) et, au-delà, la recherche de synergies entre les différents niveaux d'organisation, à travers l'imbrication nouvelle des communautés locales, dans les institutions à plus grande échelle. Ce point constitue un enjeu essentiel de la réflexion sur la place nouvelle de l'État moins surplombante que partenaire d'initiatives locales (voir Bauwens, 2014). Au-delà donc de demander la reconnaissance étatique du droit à l'auto-organisation ou d'étudier la manière dont les institutions publiques doivent soutenir et s'articuler au développement des communs, il s'agit, pour nous, de poser des jalons pour déterminer si et comment l'État peut intervenir au sein même des communautés, au nom de la conception moderne de la liberté individuelle, dans le nouveau cadre collectif que dessinent les communs.

\section{Une institution tierce pour parer aux interférences arbitraires liées au fonctionnement de petites communautés auto-organisées}

Comme nous l'avons rappelé, l'approche promue par l'essentiel des partisans des communs fait référence à l'organisation de microcommunautés auto-organisées. Dans l'approche d'E. Ostrom, le droit d'exclure que détient la communauté est essentiel à la gestion de la ressource mais il représente, en même temps, un pouvoir potentiellement arbitraire à l'égard des outsiders. Dans l'approche de Dardot et Laval, les droits, ne relevant que de l'usage subordonné à une destination sociale du bien commun, impliquent l'inclusion de la plus vaste communauté d'usagers (sans pour autant que la question de la délimitation de cette communauté ne soit sérieusement posée) ; l'exclusion (d'individus surnuméraires sur une place publique bondée, par exemple) se faisant, non pas en raison de droits d'exclure détenus par les membres de la communauté, mais au nom de finalités communes (la préservation de la place dans sa qualité de lieu ouvert à l'usage du plus grand nombre, usage compromis par une trop forte affluence). Dans les deux cas toutefois, on suppose, d'une part, que l'existence d'un commun ne relève pas de caractéristiques substantielles, mais de l'existence d'une communauté délibérante, et, d'autre part, que c'est la régulation collective qui produit, in fine, les cadres de la légitimité quant à l'exploitation d'une ressource. Or, une telle approche tend à se nourrir d'une conception abstraite et fantasmée de la communauté autant qu'à confondre communauté et démocratie.

Ainsi, il est frappant de constater que, dans la plupart des propositions étudiées, les solidarités communautaires sont excessivement valorisées sans que soient suffisamment interrogées les formes de régulation qu'elles appellent, que ce soit dans l'approche d'Ostrom où les droits de gouvernance viennent réguler l'usage des droits opérationnels, alors que la 
détermination de la nature d'une bonne gouvernance et de son fondement n'est pas sérieusement interrogée ; ou que ce soit dans l'approche de Dardot et Laval où la primauté des buts communs autodéfinis met entre parenthèses les conditions de leur définition et le risque qu'ils puissent constituer l'expression de formes de pression sociale (Durkheim, 1893). Or, d'un côté, la présupposition du caractère nécessairement bon (ou au moins meilleur que celui fondé sur des procédures dites transcendantes, c'est-à-dire ayant recours à une juridiction tierce) du contenu normatif qui émerge spontanément des fonctionnements communautaires, et, d'un autre côté, la présupposition de l'adhésion des individus aux valeurs et aux règles communautaires ont pour effet de maximiser l'adhésion spontanée des individus aux formes de vie communes, de minorer les formes d'adhésion à ces communautés sous l'effet de la pression sociale ainsi que les formes de rejet de la norme commune, laquelle peut entraver la liberté des individus.

Comment donc promouvoir les solidarités portées par l'approche des communs sans pour autant compromettre la liberté des individus ? Une première condition réside dans l'existence d'une régulation susceptible d'éviter la domination. Il s'agirait d'introduire d'une macrostructure institutionnelle, pensée comme un tiers raisonnable, chargée de l'arbitrage des litiges engageant le rapport entre une communauté auto-organisée et ses membres. Une seconde condition suppose de distinguer avec rigueur fonctionnements communautaires et fonctionnements démocratiques. Les fonctionnements démocratiques ont pour caractéristiques de mettre en place des délibérations qui sont des outils adaptés aux sociétés plurielles et complexes (Morin, 2008), dans lesquelles il s'agit de mettre en accord des intérêts et des points de vue normatifs, parfois radicalement divergents (Picavet, 2011). En revanche, les fonctionnements communautaires d'accord spontané caractérisent des sociétés de taille restreinte, où l'homogénéité sociale permet des consensus sans délibération (Durkheim, 1893 ; Tönnies, 1922). Dans le cas des fonctionnements démocratiques, les contraintes de la rationalité argumentative priment sur les points de vue hétérogènes, voire antagoniques 9 . De la sorte, ils permettent de privilégier un accord raisonnablement consenti, face aux contraintes de la pression sociale et du conformisme. Or, tant que les théories des communs ne distingueront pas entre les fonctionnements communautaires et les fonctionnements démocratiques, elles ne pourront garantir les acquis normatifs des sociétés modernes (à savoir la protection des droits fondamentaux des personnes, le respect du pluralisme, la liberté d'opinion et d'expression), en particulier en ce qui concerne les droits individuels.

En outre, négliger la pression sociale forte, typique des communautés homogènes phénomène bien connu depuis Durkheim - revient à occulter les formes d'arbitraire pouvant résulter de l'inscription des individus dans un petit groupe humain qui, quoique partiel, exerce une pression bien plus forte qu'un large collectif d'individus, porteurs d'intérêts et de points de vue différents, voire divergents. Ces formes d'arbitraire et de violence perpétrées par un groupe sur l'un de ses membres sont de deux natures.

D'une part, ces violences peuvent consister en interférence directe ou indirecte (Hobbes, 1651). Dans le premier cas, les membres de la communauté décident d'entraver, en usant de leur force, la réalisation des intentions d'un de leurs membres. Comment éviter que les rapports d'une communauté à ses membres ne se muent en formes diverses de privation de liberté ou de contrainte excessive ? Cette première question impose le recours à des instances tierces, capables d'arbitrer les conflits.

\footnotetext{
${ }^{9}$ «Les institutions de l'État de droit doivent assurer un exercice efficace de l'autonomie politique dont disposent les citoyens socialement autonomes, de telle manière que, d'une part, le pouvoir d'une volonté rationnellement formée, fondé sur la communication, puisse se manifester et prendre une forme obligatoire dans les programmes de lois, et que, de l'autre, ce pouvoir fondé sur la communication puisse, à travers l'application rationnelle et l'implémentation administrative des programmes de loi, circuler à l'échelle de la société et [...] déployer sa force d'intégration sociale » (Habermas, 1997 : 195196).
} 
Au-delà des cas d'interférence arbitraire avérée entre une communauté décisionnaire et ses membres, il existe des types, beaucoup plus insidieux, d'action de la communauté sur ses membres qui relèvent, malgré tout, de privations (ou du moins d'une diminution substantielle) de liberté. On parle alors de rapports de domination et de pression indirecte (c'est-à-dire sans violence). Comment, dans ces cas, s'assurer que les individus soient investis d'une véritable capacité (empowerment) de contestation ? Comment être certain qu'ils puissent exprimer leur désaccord (voicing) (Hirschman, 1970) sans ostracisme (comme cela pouvait être le cas dans les communautés agricoles d'Ancien Régime où les individus étaient «testés » pendant un an avant d'être définitivement intégrés à la communauté villageoise) ? Ou encore, comment garantir, a minima, une possibilité de sortie (exit) (Hirschman, 1970) de la communauté qui ne soit pas trop coûteuse en cas de conflit ? Il faudrait que soient garanties ce que Pettit (1997) a appelé des formes de «contestabilité » individuelles à l'égard de la communauté des usagers dont on ne peut sous-estimer la capacité à exercer une pression sur ses membres, c'est-à-dire des formes de domination. C'est sur ce deuxième plan aussi qu'il faut se méfier du commons washing, qui tend à considérer comme a priori bon tout ce qui se revendique du commun, au sens où tout ce qui est commun n'est pas forcément bon pour l'individu. Pour ces raisons, un tiers extérieur capable d'investir les individus d'un statut qui les garantisse contre toute domination est requis (Spitz, 2014). Il peut prendre la forme d'une institution tierce médiatrice ou arbitre offrant des possibilités de contestation ou d'exit aux individus, au sein des communautés praxiques partielles auxquelles ils appartiennent.

Ainsi, deux aspects paraissent donc essentiels. Il convient de s'assurer que les fonctionnements au sein des communautés soient des fonctionnements démocratiques respectueux de la complexité sociale et de la pluralité des points de vue, selon des procédures de rationalité délibérative. Il convient également d'éviter que la nécessaire pression communautaire ne se mue en violence ou en domination. Ces deux aspects apparaissent essentiels à intégrer à la théorie des communs, pour la compléter et en éviter son interprétation partiale (c'est-à-dire excessivement optimiste, voire idyllique) et trompeuse en matière de solidarités communautaires.

\section{Importance d'un tiers partenaire pour assurer la promotion des droits sociaux fondamentaux}

Par ailleurs, il ne suffit pas de penser les communs comme des ressources partagées, que ce soit sous la forme de droits distribués ou sous la forme d'usages subordonnés à des finalités communes, encore faut-il penser les ressources comme les vecteurs de l'accomplissement humain. En ce sens, les ressources ne doivent pas être simplement appréhendées comme des choses appropriables ou utilisables, mais aussi comme des vecteurs pour l'accomplissement de nouveaux droits sociaux fondamentaux, dont les individus seraient détenteurs (droit d'accès à l'eau potable, à une nourriture équilibrée, à la santé, à l'information, à la culture, à un environnement sain, au travail...) (Crétois, 2014 ; Crétois et Guibet Lafaye, 2015). Dans ce cadre, la question des modes d'appropriation (y compris communs) n'est pas l'enjeu essentiel, mais la possibilité pour les individus de disposer de l'accès aux ressources nécessaires à l'exercice de leurs droits sociaux fondamentaux. Il s'agit avant tout de dessiner des arrangements institutionnels susceptibles d'offrir à tous l'accès à la satisfaction de droits sociaux fondamentaux ou de capabilités fondamentales (Sen, 1985 ; Nussbaum, 2011).

Sous cet angle, les ressources sont aussi pensées comme des communs d'un type particulier qui sont moins articulés au pouvoir des communautés (comme l'entendait la définition des communs étudiée dans la première partie de cet article) qu'à la satisfaction des droits humains. C'est à ce titre que certaines ressources peuvent, y compris quand elles 
relèvent d'intérêts privés, être considérées comme appartenant au patrimoine commun de l'humanité (Boidin et al., 2008), en tant que leur usage est susceptible d'avoir des incidences non négligeables, quant à la jouissance des droits fondamentaux.

Le commun est alors pensé sur un mode distributif, à partir de la détermination de droits sociaux fondamentaux, plutôt que sur un mode collectif ou communautaire d'appropriation qui ne suffit pas à garantir l'émancipation mais qui peut, s'il n'est pas correctement encadré par une institution tierce, obérer l'émancipation. Ce statut permet de protéger des biens communs comme les ressources environnementales, non pas au nom d'un droit de telle ou telle communauté humaine, mais au nom du droit des individus de bénéficier, par exemple, d'un environnement non pollué (voir la Constitucion del Ecuador, 2008, notamment le titre II qui porte sur le buen vivir ${ }^{10}$, Crétois et Guibet Lafaye, 2015). Selon cette approche, fondée sur une régulation par les droits - plutôt que fondée sur la référence aux organisations communautaires -, il s'avère possible de penser une gouvernance collective dont le programme d'émancipation s'appuie sur l'articulation d'un individualisme normatif avec la promotion de nouvelles formes d'inclusions sociales. Cette exigence constitue alors un idéal régulateur et un principe de limitation du pouvoir décisionnel de la communauté.

Cette approche n'entrave évidemment pas le développement des communs, tels que définis dans la première partie de cet article. Elle lui est, au contraire, consubstantielle. Elle vient toutefois apporter un correctif aux biais éventuels, dont ils peuvent être l'objet, et à la régulation de leur développement. Celui-ci nécessite, à notre sens, le recours à un tiers dont le caractère transcendant est essentiel. Ce tiers constitue une instance arbitrale susceptible d'intervenir à plusieurs niveaux, en fonction d'un principe de subsidiarité, nécessaire à l'encadrement de la complexité des organisations humaines (Delsol, 2015) (le palier étatique n'étant pas toujours le plus adapté aux régulations locales). Dans ce cadre, l'institution tierce n'appartient plus au paradigme de la souveraineté, fondateur de l'État-nation. Elle tient au libre développement des communautés partielles, libre développement dont l'institution tierce assure une régulation arbitrale (visant à éviter la domination dans les relations au sein des communautés locales) ainsi que l'encadrement des fins, sans les déterminer a priori (une charte de droits sociaux fondamentaux pouvant être un de ces cadres régulateurs car, en tant que telle, elle ne détermine pas à proprement parler les fins spécifiques que doivent assumer les agents mais les limites au sein desquelles un système juste peut se déployer). L'institution tierce est alors un partenaire multiniveau (Bauwens, 2014) des plans complexes de développement social, plans irréductibles à la vision simplifiante d'une volonté universelle.

Cette approche qui, tout en se situant dans la perspective contemporaine de promotion des communs, la renouvelle substantiellement. Elle permet en effet d'éviter que les délibérations collectives ne soient orientées d'une façon qui mette à mal la protection de la liberté individuelle, tout en veillant à ce qu'elles soient guidées par l'impératif d'accomplissement humain. Cette approche éviterait également que les délibérations aient des conséquences contraires à l'impératif d'émancipation.

\section{Conclusion}

Telle que nous la concevons, une institution tierce doit être chargée de promouvoir, d'orienter et de limiter la praxis des communautés de production et de consommation partielles. Cette institution peut être - ou non - nommée État, tant il est vrai que l'organisation des institutions tierces peut se faire selon plusieurs modalités et à plusieurs niveaux, c'est-à-dire sans accorder de privilège particulier au niveau étatique, historiquement

${ }^{10}$ La Constitution de l'Équateur présente, en son deuxième chapitre, des considérations sur le bien vivre (buen vivir), traitées sous la forme de droits fondamentaux : droit à l'eau (art. 12) ; droit à une alimentation saine, suffisante et nutritive (art. 13) ; droit à un environnement sain (tit. 2, art. 14 et 15)... Constitución del ecuador (2008). 
constitué, mais en ménageant des formes de complémentarité et de subsidiarité institutionnelles à tous les étages organisationnels. Cette institution tierce macro-structurelle aurait un rôle propre qui ne saurait se réduire à celui d'une communauté englobant d'autres communautés, dans la mesure où sa fonction serait d'empêcher précisément les biais communautaires, mais également de porter l'existence d'un monde démocratique fondé sur un individualisme normatif, compatible avec de nouvelles formes d'inclusions sociales, pluraliste en matière d'émancipation humaine et qui sache intégrer conflits et désaccords par des formes de prise de décisions consensuelles ou non (Picavet, 2011) (protection contre la domination, promotion des droits fondamentaux).

\section{Bibliographie}

BAUWENS, Michel (2014), Sauver le monde, vers une société post-capitaliste avec le P2P, Paris, Les liens qui libèrent.

BoIDIN, Bruno, Bertrand HIEZ et Sandrine RousseAu (coord.) (2008), « Biens communs, biens publics mondiaux et propriété », Développement durable et territoires, Dossier $10 \mid$ 2008 : Biens communs et propriété. En ligne à https://developpementdurable.revues.org/5143, consulté le 22 juin 2015.

CAILle, Alain (2011), Pour un manifeste du convivialisme, Lormont, Le Bord de l'Eau.

Chanteau, Jean-Pierre et Agnès LABrousse (2013) «L'institutionnalisme méthodologique d'Elinor Ostrom : quelques enjeux et controverses », Revue de la régulation, $14 \mid 2^{\mathrm{e}}$ semestre / Automne 2013. En ligne à http://regulation.revues.org/10555, consulté le 30 juin 2015 .

Chappe, Raphaël et Pierre Cretois (dir.) (2014), L’homme présupposé, Aix-en-Provence, Presses universitaires de Provence.

Constitución del ecuador (2008). Political Database of the Americas, Georgetown University, Center for Latin American Studies. En ligne à http://pdba.georgetown.edu/Parties/Ecuador/Leyes/constitucion.pdf, consulté le 5 janvier 2016.

CORIAT, Benjamin (2015a), «Qu'est-ce qu'un commun ? Quelles perspectives le mouvement des communs ouvre-t-il à l'alternative sociale ? », Les possibles, J.-M. HARRIBEY et J. TosTI (dir.), dossier $n^{0} 5$ : Les biens communs. En ligne à https://france.attac.org/nospublications/les-possibles/numero-5-hiver-2015/dossier-les-biens-communs/article/qu-est-cequ-un-commun, consulté le 5 janvier 2016.

CORIAT, Benjamin (2015b), Le retour de communs, Paris, Les liens qui libèrent.

COUTURE, Stéphane (2013), «Logiciels libres : réduction des coûts et souveraineté numérique », Institut de recherche et d'information socio-économique, note du 20 septembre. En ligne à http://iris-recherche.s3.amazonaws.com/uploads/publication/file/Note-Logicielslibres.pdf, consulté le 5 janvier 2016.

CRETOIS, Pierre (2014), «La propriété repensée par l'accès », Revue internationale de droit économique, 2014/3, t. XXVIII, p. 319-334.

CRETOIS, Pierre (2015), Le renversement de l'individualisme possessif de Hobbes à l'État social, Paris, Les classiques Garnier.

CRETOIS, Pierre et Caroline GuIBET LAFAYE (2015), « Y a-t-il une alternative à la conception exclusiviste classique du droit de propriété ? Une théorie inclusive de 
l'appropriation », dans Pierre CRETOIS et Thomas BoCCON-GIBOD (dir.), État social, propriété publique et biens communs, Bordeaux, Le Bord de l'eau.

DagAn, Hanoch et Michaël A. Heller (2001), «The liberal commons », Yale Law Journal, vol. 110.

Dardot, Pierre et Christian Laval (2014), Commun. Essai sur la révolution au XXI siècle, Paris, La Découverte.

DeLsol, Chantal (2015), L'État subsidiaire, Paris, Lexio.

DURKHEIM, Émile ([1893] 2007), De la division du travail social, Paris, Presses universitaires de France.

Flahault, François (2011), Où est passé le bien commun ?, Paris, Mille et une nuits.

Follain, Antoine (2008), Le village sous l'Ancien Régime, Paris, Fayard.

GuiBet LAFAYE, Caroline (2016), «Quel modèle pour penser l'inappropriable ? », Revue de philosophie économique, Aix-en-Provence.

HABERMAS, Jürgen (1997), Droit et démocratie - Entre faits et normes, Paris, Gallimard.

HARDIN, Garrett (1968), «The Tragedy of the Commons », Science, vol. 162, n 3859, p. 1243-1248.

HARRIBEY, Jean-Marie (dir.) (2015), « Pour une conception matérialiste des biens communs », Les possibles, J.-M. HARRIBEY et J. TOSTI (dir.), dossier $n^{\circ} 5$ : Les biens communs. En ligne à https://france.attac.org/nos-publications/les-possibles/numero-5-hiver2015/dossier-les-biens-communs/article/pour-une-conception-materialiste-des-bienscommuns, consulté le 5 janvier 2016.

Hirschman, Albert (1970), Exit, Voice, and Loyalty: Responses to Decline in Firms, Organizations, and States, Cambridge, MA, Harvard University Press.

HobBes, Thomas ([1651] 1999), Léviathan, Paris, Dalloz.

HOLland, Guillaume et Omar SENE (2010), «Elinor Ostrom et la Gouvernance économique », Revue d'économie politique, 2010/3, vol. 120, p. 441-452. En ligne à www.cairn.info/revue-d-economie-politique-2010-3-page-441.htm, consulté le 5 janvier 2016.

MichEA, Jean-Claude (2007), L'empire du moindre mal. Essai sur la civilisation libérale, Paris, Flammarion.

MORIN, Edgar (2008), La complexité humaine, Paris, Flammarion.

NeGRI, Antonio et HARDT, Michaël (2011), Commonwealth London, Belknap Press.

Nussbaum, Martha C. (2011), Capabilités, Coment créer les conditions d'un monde plus juste?, traduction de S. Chavel, Paris, Climats.

ORSI, Fabienne (2015), «Reconquérir la propriété : un enjeu déterminant pour l'avenir des communs », Les possibles, J.-M. HARRIBEY et J. TOSTI (dir.), dossier $\mathrm{n}^{\circ} 5$ : Les biens communs. En ligne à https://france.attac.org/nos-publications/les-possibles/numero-5-hiver2015/dossier-les-biens-communs/article/reconquerir-la-propriete, consulté le 5 janvier 2016.

Ostrom, Elinor (1990), Governing the Commons. The Evolution of Institutions for Collective Action, Cambridge et New York, Cambridge University Press. 
OStRoM, Elinor et Edella Schlager (1992), «Property-rights regimes and natural resources: a conceptual analysis », Land and economics, vol. 68, $\mathrm{n}^{\mathrm{0}} 3$, University of Wisconsin Press, p. 249-262.

PARANCE, Béatrice et Jacques DE SAINT VICTOR (2014), Repenser les biens communs, Paris, CNRS Éditions.

PetTit, Philip (1887), Républicanisme. Une théorie de la liberté et du gouvernement, édition française, Paris, Gallimard.

PicAVet, Emmanuel (2011), La revendication des droits: Une étude de l'équilibre des raisons dans le libéralisme, Paris, Les classiques Garnier.

RAWLS, John ([1971] 1987), Théorie de la justice, édition française, Paris, Seuil.

Samuelson, Paul A. (1954), «The Pure Theory of Public Expenditure », Review of Economics and Statistics, vol. 36, n 4, p. 387-389.

SAUVETRE, Pierre (2015), «Foucault et le conflit démocratique : le gouvernement du commun contre le gouvernement néolibéral », Astérion, Dossier 13|2015 : La démocratie à l'épreuve du conflit. En ligne à http://asterion.revues.org/2648, consulté le 9 juin 2015.

SEn, Amartya (1985), Commodities and Capabilities, Amsterdam, North-Holland.

SPITZ, Jean-Fabien (2014), Le mythe de l'impartialité, Paris, Presses universitaires de France.

TöNNIES, Ferdinand ([1922] 2010), Communauté et société, Paris, Presses universitaires de France.

WeINSTEIN, Olivier (2015), «Comment se construisent les communs : questions à partir d'Ostrom », dans Benjamin CORIAT (dir.), Le retour des communs, Paris, Les liens qui libèrent, p. 69-86. 